\title{
Una implementación abierta y generalizada del método predictivo de Soave-Redlich-Kwong para el cálculo de la constante de la ley de Henry
}

\author{
Sebastián Duque-Tobón*, Javier I. Carrero-Mantilla*§ \\ * Universidad Nacional de Colombia, Sede Manizales, Manizales, Colombia \\ $\S$ e-mail: jicarrerom@unal.edu.co
}

(Recibido: Abril 03 de 2008 - Aceptado: Octubre 30 de 2008)

\section{Resumen}

La predicción de la solubilidad de gases en líquidos utilizando la ley de Henry es un problema importante en termodinámica química por sus aplicaciones a procesos industriales de separación gas-líquido. Sin embargo, el cálculo de la constante de la ley de Henry mediante ecuaciones de estado requiere implementaciones computacionales que son difíciles de programar en un formato generalizado. Por estar basado en el modelo UNIFAC de coeficientes de actividad, el método predictivo de Soave-Redlich-Kwong (PSRK) se puede aplicar a numerosos sistemas de interés. Considerando estas razones, se desarrolló una implementación abierta y generalizada del método PSRK para su aplicación al cálculo de la constante de la ley de Henry en el equilibrio gas-líquido. Esta implementación se puso a prueba al comparar las predicciones del método PSRK con un conjunto reciente de mediciones experimentales de constantes de la ley de Henry en sistemas de compuestos orgánicos. Se encontró que, dentro de un amplio rango de temperaturas, las constantes de la ley de Henry obtenidas mediante el método PSRK concuerdan razonablemente bien con los resultados experimentales para la mayor parte de los sistemas incluidos en la comparación.

Palabras clave: Ley de Henry, Método PSRK, Solubilidad de gases, Equilibrio gas-líquido.

CHEMICAL ENGINEERING

\section{An open and generalized implementation of the Soave-Redlich-Kwong predictive method for the calculation of Henry's law constant}

\begin{abstract}
The prediction of the solubility of gases in liquids by use of Henry's law is an important problem in chemical thermodynamics because of its applications to industrial gas-liquid separation processes. However, the calculation of Henry's law constant by means of equations of state requires computational implementations that are difficult to program in a generalized format. Because of its basis on the UNIFAC model of activity coefficients, the predictive Soave-Redlich-Kwong (PSRK) method can be applied to numerous systems of interest. From the consideration of these reasons, an open and generalized implementation of the PSRK method was developed for its application to the calculation of Henry's law constant in gas-liquid equilibrium. This implementation was tested by comparing the predictions of the PSRK method for Henry's law constant with a recent set of experimental measurements in systems of organic compounds. It was found that, within a wide temperature range, Henry's law constants obtained from the PSRK method agree reasonably well with the experimental results for most of the systems included in the comparison.
\end{abstract}

Keywords: Henry's law, PSRK method, Gas solubility, Gas-liquid equilibrium. 


\section{Introducción}

La ley de Henry relaciona la fugacidad parcial $\hat{f}_{i}$ de un soluto $i$ con su fracción molar $x_{i}$, usando una constante de proporcionalidad $H_{i, j}$ llamada constante de la ley de Henry, la cual depende del soluto $(i)$ y el solvente $(j)$ :

$$
\hat{f}_{i}=x_{i} H_{i, j}
$$

Como permite el uso de diferentes estados de referencia para las fugacidades parciales de los componentes de una solución, la ley de Henry es válida para casos en los que el soluto no puede existir como líquido a las condiciones de la mezcla. Esto la hace muy útil para estimar la solubilidad de gases en líquidos: algunas de sus aplicaciones están en el diseño de procesos de absorción, análisis ambiental, modelos de gases disueltos en agua o soluciones acuosas, y geoquímica. Dado que la ley de Henry es válida usualmente a bajas fracciones molares del soluto, la constante $H_{i, j}$ se puede definir como

$$
H_{i, j}=\lim _{x_{i} \rightarrow 0} \frac{\hat{f_{i}}}{x_{i}}
$$

para un sistema solvente-soluto $(i, j)$ a una temperatura dada. Una simplificación muy común a bajas presiones es reemplazar la fugacidad parcial del soluto $i$ por su presión parcial, de modo que la Ec.(2) se convierte en

$$
H_{i, j}=\lim _{x_{i} \rightarrow 0} \frac{P_{i}}{x_{i}}
$$

La Ec. (2) se evalúa a la presión de saturación del solvente porque

$$
\lim _{x_{i} \rightarrow 0} P=P_{j}^{\mathrm{sat}}
$$

y así $H_{i, j}$ depende de la temperatura $T$ porque $P_{j}^{\text {sat }}$ es función de la temperatura. Sin embargo, el cociente $\hat{f}_{i} / x_{i}$ se puede extrapolar a presiones distintas a $P_{j}^{\text {sat }}$ a partir de la relación

$$
\left[\frac{\partial \ln \left(\hat{f}_{i}\right)}{\partial P}\right]_{T, x}=\frac{\bar{V}_{i}}{R T}
$$

en la cual se basa la ecuación de KrichevskyKasarnovsky descrita por Prausnitz et al. (2000):

$$
\ln \left(\frac{\hat{f_{i}}}{x_{i}}\right)_{P}=\ln H_{i, j}+\frac{\bar{V}_{i}^{\infty}}{R T}\left(P-P_{j}^{\mathrm{sat}}\right)
$$

donde $\bar{V}_{i}^{\infty}$ es el volumen molar parcial del soluto $i$ en la solución en el límite de dilución infinita.

La medición experimental es la fuente primaria de valores de la constante de la ley de Henry. Con la simplificación que acarrea la Ec. (3), los valores de $H_{i, j}$ se pueden estimar indirectamente a partir de datos de equilibrio líquido-vapor $\left(P, x_{i}, y_{i}\right)$ en el límite $x_{i} \rightarrow 0$, que coincide con la región de baja presión. También hay procedimientos experimentales diseñados para obtener directamente $H_{i, j}$, como el método de despojamiento de gas descrito por Leroi et al. (1977). Si bien hay datos publicados de $H_{i, j}$ para solventes industriales, como el gasóleo (Ronze et al., 2002), y para sistemas de interés ambiental, como por ejemplo, contaminantes disueltos en agua (Jantunen \& Bidleman, 2006), muchas de las mediciones de $H_{i, j}$ reportadas en la literatura se refieren a sistemas solvente-soluto de interés químico, por ejemplo, las reportadas por Miyano et al. (2003a, 2003b), Miyano (2004a, 2004b, 2004c, 2004d), Miyano (2005a, 2005b, 2005c), Miyano et al. (2005d), y Miyano et al. (2006a, 2006b).

La simulación molecular es otra fuente de valores de $H_{i, j}$, que se puede calcular como el producto de dos contribuciones:

$$
H=H^{\text {id }} H^{\mathrm{r}}
$$

una contribución de gas ideal (id),

$$
H^{\text {id }}=\rho_{j} R T
$$

y una contribución residual (r),

$$
H^{\mathrm{r}}=\exp \left[\frac{\mu_{i}^{\mathrm{r}, \infty}}{R T}\right]
$$


Con la Ec. (7), se pueden estimar valores de $H_{i, j}$ basados exclusivamente en modelos moleculares, sin necesidad de información experimental: la densidad se obtiene a partir de simulaciones a presión constante, $\mathrm{y} \mu_{i}^{\mathrm{r}, \infty}$ se obtiene por medio del método de inserción de moléculas (Widom, 1963), o alguna de sus variantes; aunque también hay métodos de simulación de dinámica molecular para calcular $H_{i, j}$ que no requieren inserciones (Murad \& Gupta, 2000; Murad \& Gupta, 2001). El interés en los métodos de simulación molecular ha aumentado recientemente; por ejemplo, la estimación de $H_{i, j}$ fue uno de los problemas propuestos en la segunda edición (2003-2004) del Industrial Fluid Properties Simulation Challenge (IFPS, 2008), que ha generado numerosas publicaciones: Cichowski et al. (2005), Schnabel et al. (2005), Wu et al. (2005), Zhang \& Siepmann (2006).

Los métodos experimentales y de simulación molecular proporcionan datos de $H_{i, j}$ de primera mano, pero tienen desventajas. Los métodos experimentales requieren montajes que pueden ser muy costosos y la normalización de los procedimientos. En comparación, las simulaciones moleculares pueden ser relativamente baratas, pero requieren códigos computacionales especializados que necesitan ser ajustados para cada sistema solvente-soluto, y además los métodos tienen limitaciones, por ejemplo, la inserción de moléculas con el método de Widom requiere un alto número de inserciones para obtener una estadística aceptable de los resultados, de forma que se necesitan corridas de simulación bastante largas. Por eso, en cálculos prácticos de ingeniería puede ser preferible utilizar representaciones generalizadas de las fases líquida y gaseosa: modelos de coeficientes de actividad (MCA) y ecuaciones de estado (EOS).

El equilibrio de fases gas-líquido implica la igualdad de las fugacidades parciales del soluto gaseoso $i$ en ambas fases. Así que $\hat{f}_{i}^{G}=\hat{f}_{i}^{L}$, pero la forma de calcular $\hat{f}_{i}$ es distinta: mientras que en el gas se usa el coeficiente de fugacidad parcial $\hat{\varphi}_{i}{ }^{G}$ a través de la expresión $\hat{f}_{i}^{G}=\hat{\varphi}_{i}^{G} y_{i} P$, en el líquido se aplica la ley de Henry según la Ec. (1), $\hat{f}_{i}^{L}=x_{i} H_{i, j}$. De esta forma, se tiene que:

$$
\hat{\varphi}_{i}^{G} y_{i} P=x_{i} H_{i, j}
$$

y el coeficiente de fugacidad parcial $\hat{\varphi}_{i}^{G}$ se puede obtener mediante una ecuación de estado como una función de $T, P$ y las composiciones $y_{i}$. Sin embargo, este método requiere suministrar valores de $H_{i, j}$ para calcular la solubilidad $x_{i}$ de $i$ en la fase líquida. Pero en lugar de usar la Ec. (10), es posible calcular $H_{i, j}$ directamente a partir de una ecuación de estado aplicada a la fase líquida, reemplazando $\hat{f}_{i}=x_{i} \hat{\varphi}_{i}^{L} P$ en la Ec. (2) para obtener la forma general:

$$
H_{i, j}=\lim _{x_{i} \rightarrow 0}\left(\hat{\varphi}_{i}^{L} P\right)
$$

Para ello, $\hat{\varphi}_{i}^{L}$ se obtiene aplicando la ecuación de estado a la fase líquida, y como la presión $P$ en el límite $x_{i} \rightarrow 0$ tiende a la presión de vapor del solvente $P_{j}^{\text {sat }}$, esta última también se calcula con la ecuación de estado. El método de la Ec. (11) resulta ser una opción muy apropiada para cálculos de ingeniería, siempre y cuando se cuente con una ecuación de estado que sea predictiva para el sistema solvente-soluto $i-j$.

Se ha encontrado que el uso de EOS cúbicas, por ejemplo, las ecuaciones de Peng-Robinson (PR) y Soave-Redlich-Kwong (SRK) con reglas de mezclado convencionales para calcular $H_{i, j}$, requiere parámetros de interacción binarios específicos para cada par soluto-solvente, más cuando las moléculas de los componentes del sistema tienen tamaños muy distintos (Bader \& Gasem,1996). Esto sugiere que puede ser ventajoso utilizar métodos predictivos que no dependan de parámetros de interacción binarios, en particular, los métodos que utilizan MCA para cuantificar las interacciones $i-j$ (llamados métodos $\hat{G}^{\text {ex }}-$ EOS ). Pero los métodos $\hat{G}^{\text {ex }}-$ EOS pueden tener también dificultades para predecir $H_{i, j}$. Las capacidades predictivas de tres modelos $\hat{G}^{\text {ex }}$ - EOS: LVCM de Boukouvalas et al. (1994), MHV2 de Dahl et al. (1990, 1991) y PSRK de Holderbaum \& Gmehling (1991) fueron comparadas para un conjunto grande de sistemas gas-líquido, incluyendo los solutos $\mathrm{O}_{2}, \mathrm{~N}_{2}, \mathrm{CO}_{2}$, $\mathrm{CO}, \mathrm{H}_{2} \mathrm{~S}, \mathrm{CH}_{4}, \mathrm{C}_{2} \mathrm{H}_{6}, \mathrm{C}_{3} \mathrm{H}_{8}$ y $\mathrm{C}_{4} \mathrm{H}_{10}$ disueltos en cetonas, alcoholes, n-alcanos y agua por Apostolou et al. (1995), y se encontró que el 
modelo LVCM daba los mejores resultados, mientras que PSRK tenía dificultades, especialmente en sistemas asimétricos, aunque sus resultados eran comparables con los del modelo MHV2. Sin embargo, hay argumentos que justifican elegir al modelo PSRK para calcular $H_{i, j}$ : ha resultado satisfactorio para predecir solubilidades de gases, su base de datos de parámetros de interacción ha sido corregida y extendida sin interrupción, y es accesible públicamente (Gmehling et al., 1997; Horstmann et al., 2005; Li et al.,1998).

En los simuladores de procesos se pueden encontrar bases de datos que incluyen información de los componentes más comunes y métodos predictivos para $H_{i, j}$. Sin embargo, este tipo de paquetes computacionales tienden a ser muy costosos, y también cerrados porque restringen el acceso del usuario a los códigos de los cálculos y a los valores almacenados en sus bases de datos. Para propósitos didácticos y también para aplicaciones prácticas de ingeniería, resulta de gran valor contar con un método predictivo que pueda ser modificado o adaptado por el usuario. Por eso, en este trabajo se expone la construcción y prueba de un conjunto de funciones que permiten a cualquier usuario calcular $H_{i, j}$ y también hacer cálculos de equilibrios de fases con el modelo PSRK y su base de datos pública. El resto de este artículo está organizado así: en la Sección 2 se presentan las reglas de mezclado necesarias para la aplicación de una ecuación de estado a un sistema multicomponente y se explica la ventaja de una formulación predictiva como la del método PSRK. En la Sección 3, se explica el cálculo de $H_{i, j}$ con el método PSRK, y en la Sección 4 se explica la implementación y se muestran resultados para un conjunto amplio de sistemas y su comparación con los datos experimentales.

\section{Metodología}

\subsection{El método PSRK}

Para los cálculos típicos de ingeniería química, ha prevalecido el uso de dos EOS cúbicas basadas en la ecuación de estado de van der Waals: la ecuación de estado PR (Peng \& Robinson,1976) y la ecuación de estado SRK (Soave et al.,1972), debido a sus buenas capacidades predictivas y relativa sencillez, aunque se reconoce que su predicción de las densidades de líquido no siempre es buena. La ecuación de estado SRK,

$$
P=\frac{R T}{\hat{V}-b}-\frac{a(T)}{\hat{V}(\hat{V}+b)}
$$

es una modificación de la ecuación de estado de Redlich-Kwong (Redlich \& Kwong,1949), que incluye la forma del parámetro atractivo $a(T)$ modificada por Soave:

$$
a(T)=a f(T)
$$

donde $a$ y $b$ dependen de las propiedades críticas, así:

$$
\begin{aligned}
& a=0.42748 \frac{\left(R T_{c}\right)^{2}}{P_{c}} \\
& b=0.08664 \frac{R T_{c}}{P_{c}}
\end{aligned}
$$

y la función $f$ depende de la temperatura reducida, así:

$$
f(T)=\left[1+c_{1}\left(1-T_{r}^{0,5}\right)\right]^{2}
$$

con

$$
T_{r}=\frac{T}{T_{c}}
$$

y

$$
c_{1}=0.48+1.574 \omega-0.176 \omega^{2}
$$

En términos del factor de compresibilidad,

$$
Z=\frac{P \hat{V}}{R T}
$$

y con los parámetros adimensionales

$$
A=0.42748\left[\frac{P_{r}}{\left(T_{r}\right)^{2}}\right] f(T)
$$


donde $P_{r}=P / P_{c} \quad$ y

$$
B=0.08664\left(\frac{P_{r}}{T_{r}}\right)
$$

La Ec. (12) se puede escribir como una ecuación cúbica, así:

$$
Z^{3}-Z^{2}+\left(A-B-B^{2}\right) Z-A B=0
$$

Esta ecuación cúbica es muy conveniente porque las raíces reales de la Ec. (22) corresponden a los factores de compresibilidad de la(s) fase(s) que existen a $T$ y $P$ especificados. Pero para aplicar la Ec. (22) a la predicción de $Z$ para una mezcla, resulta necesario utilizar reglas de mezclado para obtener $a$ y $b$ (o $A$ y $B$ ) de la mezcla como un todo, porque cada componente de la mezcla tiene sus propias propiedades críticas. Las reglas de mezclado más comunes son las de un fluido de van der Waals:

$$
\begin{gathered}
a=\sum_{i} \sum_{j} x_{i} x_{j} a_{i j} \\
b=\sum_{i} \sum_{j} x_{i} x_{j} b_{i j}
\end{gathered}
$$

con parámetros cruzados $a_{i j}$ y $b_{i j}$ que dependen a su vez de parámetros de interacción binarios $k_{i j}$ y $l_{i j}$ según las ecuaciones

$$
a_{i j}=\left(1-k_{i j}\right) \sqrt{a_{i} a_{j}}
$$

$\mathrm{y}$

$$
b_{i j}=\frac{1}{2}\left(1-l_{i j}\right)\left(b_{i}+b_{j}\right)
$$

Según Orbey \& Sandler (1998), las reglas de mezclado de van der Waals se clasifican por el número de parámetros de interacción binarios efectivamente usados en las Ecs. (25) y (25): si sólo se emplea el parámetro $k_{i j}\left(\right.$ dejando $\left.l_{i j}=0\right)$ la regla de mezclado es 1PVDW (one parameter van der Waals); mientras que a las reglas de mezclado no cuadráticas en las que el parámetro $k_{i j}$ depende de la composición, dejando la regla de mezclado para $b$ sin cambiar, se les denomina 2PVDW (aunque también sería factible llamar 2PDVW a una regla de mezclado que incluyera dependencia con $k_{i j}$ y $l_{i j}$ ). El enfoque convencional con las reglas de mezclado 1PVDW o 2PVDW puede dar buenos resultados para el equilibrio gas-líquido, pero requiere para cada sistema el ajuste de datos experimentales, incluso respecto a la temperatura, para obtener la mejor capacidad predictiva de la ecuación de estado mediante $k_{i j}$ o $l_{i j}$ y en todo caso, los valores de estos parámetros son válidos sólo para un par especifico $i-j$ (Bader \& Gasem,1996; Mullins et al.,1998; Sieder $\&$ Maurer, 2004).

Para superar las limitaciones de las reglas de mezclado 1PVDW o 2PVDW se ha propuesto cuantificar las interacciones intermoleculares usando un modelo de coeficientes de actividad (MCA) en lugar de los parámetros de interacción binaria. La ecuación de estado (EOS) se acopla al MCA utilizando la igualdad de la energía libre de Helmholtz o la energía libre de Gibbs. Como $\hat{G}^{\text {ex }}$ se relaciona con los coeficientes de actividad a través de la expresión:

$$
\frac{\hat{G}^{\mathrm{ex}}}{R T}=\sum_{i} x_{i} \ln \gamma_{i}
$$

entonces se puede igualar el valor $\hat{G}_{\gamma}^{\text {ex }}$ dado por la Ec. (27) y el valor obtenido con una EOS, así:

$$
\hat{G}_{\gamma}^{\mathrm{ex}}=\hat{G}_{\mathrm{EOS}}^{\mathrm{ex}}
$$

de forma que los parámetros $a$ y $b$ de la mezcla dependen de los conjuntos $\left\{a_{i}\right\}$ y $\left\{b_{i}\right\}$ y a su vez dependen de la temperatura y la composición a través del MCA. De esta igualación, proviene la denominación $\hat{G}^{\mathrm{ex}}-\mathrm{EOS}$. La igualdad en la Ec. (28) puede aplicarse también a $\hat{A}_{y}^{\text {ex }}$ pero $\hat{A}^{\text {ex }}$ o $\hat{G}^{\text {ex }}$ obtenidos a partir de una EOS dependen de $P$, mientras que $\hat{G}^{\text {ex }}$ obtenida de un MCA no depende de $P$, lo que implica que la igualdad de $\hat{A}^{\text {ex }}$ o $\hat{G}^{\text {ex }}$ debe hacerse a un valor definido de presión (Orbey \& Sandler, 1998). Por eso, los modelos se pueden clasificar según el tipo de igualdad, así:

$\hat{G}_{\mathrm{EOS}}^{\mathrm{ex}}\left(T, P \rightarrow \infty,\left\{x_{i}\right\}\right)=\hat{G}_{\gamma}^{\mathrm{ex}}\left(T, P \rightarrow \infty,\left\{x_{i}\right\}\right)$

(Huron \& Vidal, 1979), y

$\hat{A}_{\text {EOS }}^{\text {ex }}\left(T, P \rightarrow \infty,\left\{x_{i}\right\}\right) \approx \hat{G}_{\gamma}^{\text {ex }}\left(T, P_{\text {baja }},\left\{x_{i}\right\}\right)$

(Wong et al., 1992), 
$\hat{G}_{\mathrm{EOS}}^{\mathrm{ex}}\left(T, P=0,\left\{x_{i}\right\}\right)=\hat{G}_{\gamma}^{\mathrm{ex}}\left(T, P=0,\left\{x_{i}\right\}\right)$

(Dahl et al., 1990; Michelsen,1990).

Los modelos $\hat{G}^{\text {ex }}-$ EOS necesitan un modelo de coeficientes de actividad, pero los parámetros de los modelos usuales, por ejemplo, ScatchardHildebrand o NRTL deben ajustarse a partir de información experimental de las mezclas específicas, que no siempre está disponible. Para evitar esta dificultad en la obtención de parámetros y construir modelos $\hat{G}^{\mathrm{ex}}-\mathrm{EOS}$ completamente predictivos, se pueden usar métodos de contribución de grupos, que consideran a los componentes de una solución como el resultado de la asociación de un número reducido de grupos funcionales, y que por lo tanto no dependen de ajuste de datos experimentales de mezclas. De los modelos de coeficientes de actividad basados en contribución de grupos (UNIFAC, ASOG, y sus variantes) posiblemente UNIFAC es el más extendido, pero, igual que sus contrapartes, no incluye grupos funcionales para gases porque en principio fue diseñado para soluciones líquidas.

La ecuación de estado PSRK se basa en la ecuación de estado SRK, Ecs. (12) y (13), pero en lugar de la forma original de $f$ [Ec. (16)] se utiliza la expresión de Mathias y Copeman,

$f(T)= \begin{cases}{\left[1+c_{1}\left(1-T_{r}^{0,5}\right)+c_{2}\left(1-T_{r}^{0,5}\right)^{2}+c_{3}\left(1-T_{r}^{0,5}\right)^{3}\right]^{2}} & T_{r}<1 \\ {\left[1+c_{1}\left(1-T_{r}^{0,5}\right)^{2}\right],} & T_{r}>1\end{cases}$

porque el uso de los tres parámetros ajustables $c_{1}$, $c_{2}$ y $c_{3}$ mejora especialmente la capacidad de predecir presiones de vapor de compuestos polares. La regla de mezclado para $b$ se basa en las Ecs. (24) y (26) ( con $\left.l_{i j}=0\right)$ :

$$
B=\sum_{i} x_{i} B_{i}
$$

donde $B$ es el parámetro de la mezcla como un todo y los $B_{i}$ son los parámetros de los componentes individuales dados por la Ec. (21).
La regla de mezclado para el parámetro $A$ es:

$$
\frac{A}{B}=\sum_{i} x_{i} \frac{A_{i}}{B_{i}}+\frac{1}{\Lambda}\left[\frac{\hat{G}^{\mathrm{ex}}}{R T}+\sum_{i} x_{i} \ln \left(\frac{B}{B_{i}}\right)\right]
$$

$\operatorname{con} \Lambda=\ln (1.1 / 2.1)=-0.6466271649$ (Fischer $\&$ Gmehling, 1996). El coeficiente de fugacidad parcial del componente $i$ se calcula con $A$ y $B$, así:

$$
\ln \left(\hat{\varphi}_{i}\right)=\frac{B_{i}}{B}(Z-1)-\ln (Z-B)-\bar{\alpha}_{i} \ln \left(1+\frac{B}{Z}\right)
$$

y el parámetro $\bar{\alpha}_{i}$ se relaciona con el coeficiente de actividad de $i$ :

$\bar{\alpha}_{i}=\frac{1}{\Lambda}\left[\ln \left(\gamma_{i}\right)+\ln \left(\frac{B}{B_{i}}\right)+\frac{B_{i}}{B}-1\right]+\frac{A_{i}}{B_{i}}$

El término $\hat{G}^{\mathrm{ex}} / R T$ en la Ec. (31) se calcula con el método UNIFAC,

$$
\frac{\hat{G}_{\gamma}^{\mathrm{ex}}}{R T}=\sum_{i} x_{i} \ln \left(\gamma_{i}\right)
$$

en el que el coeficiente de actividad depende de dos contribuciones, una combinatorial $(C)$, y otra residual $(R)$,

$$
\ln \left(\gamma_{i}\right)=\ln \left(\gamma_{i}^{C}\right)+\ln \left(\gamma_{i}^{R}\right)
$$

La idea central de UNIFAC es reducir la información de equilibrio de fases a parámetros de interacción entre grupos estructurales, siguiendo el concepto original de Langmuir de que las interacciones físicas entre moléculas pueden estimarse sumando interacciones entre los grupos funcionales que las forman (Fredenslund et al.,1975; Fredenslund et al., 1977a; Fredenslund et al.,1977b). Los grupos estructurales se eligen por conveniencia, por ejemplo, $\mathrm{CH}_{3} \mathrm{o}-\mathrm{COCH}_{2}-$, y los parámetros entre grupos se pueden usar para predecir coeficientes de actividad en sistemas en 
los que no hay información experimental disponible. El término combinatorial $\ln \left(\gamma_{i}^{c}\right)$, representa la contribución entrópica y depende de las fracciones relativas de volumen y superficie de las moléculas, que se calculan con los parámetros de volumen y área superficial de los grupos funcionales. El término residual $\ln \left(\gamma_{i}^{R}\right)$ representa la contribución entálpica y depende de una suma de contribuciones de cada grupo en la solución, menos la suma de las contribuciones individuales en el entorno del compuesto puro (las contribuciones de los grupos a los coeficientes de actividad dependen solamente de la temperatura y de las fracciones molares de los grupos).

En el método PSRK, el término $\hat{G}_{\gamma}^{\text {ex }}$ no ha sido concebido como un reemplazo o una extensión del término original en el modelo UNIFAC (Fredenslund et al.,1977b). En PSRK se han incluido grupos funcionales que representan gases, y como sus parámetros de interacción se obtuvieron por ajuste de datos de ELV en un rango mucho más amplio que el de UNIFAC original, entonces se incluyeron dos parámetros adicionales en la función $\Psi$ que describe la interacción entre grupos,

$$
\Psi_{n m}=\exp \left[-\frac{\left(a_{n m}+b_{n m} T+c_{n m} T^{2}\right)}{T}\right]
$$

en contraste con el método UNIFAC original, en el cual $\Psi$ sólo depende de un parámetro, $a_{m n}$. De esta forma, PSRK permite predecir el equilibrio líquido-vapor o líquido-gas para un gran número de sistemas sin necesidad de recurrir al ajuste de datos experimentales de las mezclas (Holderbaum \& Gmehling, 1991). La base de datos de parámetros de interacción de PSRK, aparte de los valores provenientes de UNIFAC, ha sido obtenida por ajuste de datos experimentales de equilibrio líquido-vapor y solubilidad de gases en líquidos (pero no de constantes de Henry $H_{i, j}$ ) provenientes de la base de datos Dortmund Data Bank, que es posiblemente la fuente más completa de datos experimentales disponible a la fecha (Fischer \& Gmehling,1996; Gmehling et al., 1997; Horstmann et al., 2000; Horstmann et al., 2005).

En diversos trabajos de desarrollo del método PSRK se han presentado nuevos parámetros para gases ligeros que permiten la predicción de equilibrios líquido-vapor y solubilidades de gases, a altas y bajas temperaturas, incluso en sistemas con compuestos polares y mezclas asimétricas, como lo describen Chen et al. (2002), Fischer \& Gmehling (1996), Gmehling et al. (1997),
Holderbaum \& Gmehling (1991), Horstmann et al. (2000), Horstmann et al. (2005) y Li et al. (1998). En el desarrollo del método PSRK se han corregido numerosos parámetros de interacción entre grupos, por ejemplo, entre compuestos aromáticos y metanol, obteniéndolos de datos experimentales que cubren un rango más amplio de presiones (Holderbaum \& Gmehling, 1991). También se ha logrado un buen ajuste de datos experimentales en sistemas polares, incluyendo puntos críticos y azeotrópicos (Fischer \& Gmehling, 1996) y permitiendo la predicción de solubilidades de gases a altas y bajas presiones (Gmehling et al., 1997; Li et al., 1998). La matriz de parámetros del método PSRK se ha actualizado hasta cubrir 1000 compuestos (Horstmann et al., 2000; Horstmann et al., 2005).

Como esos parámetros no se han obtenido directamente del ajuste de datos de constantes de la ley de Henry, el cálculo de $H_{i, j}$ es una prueba muy exigente de la capacidad predictiva del método PSRK porque los valores de $\hat{\varphi}_{i}^{L}$ en la Ec. (11) dependen tanto de las interacciones soluto-solvente cuantificadas con $\hat{G}^{\text {ex }} / R T$, como de la densidad del líquido, que se obtiene de la ecuación de estado cúbica sin correcciones de volumen adicionales. Los resultados han mostrado que la predicción de $H_{i, j}$ de gases ligeros (hidrógeno en agua, nitrógeno y oxígeno en 1-propanol) es posible con el método PSRK (Apostolou et. al.,1995; Horstmann et al, 2000). En el trabajo de Apostolou et. al. (1995), que incluyó un conjunto de 9 gases en una amplia gama de solventes, se encontraron resultados que también respaldan la elección del método PSRK para calcular $H_{i, j}$.

\subsection{Cálculo de la constante de la ley de Henry}

El primer paso para estimar $H_{i, j}(T)$ con la Ec. (11) es el cálculo de la presión de saturación del solvente $\left(P_{j}^{\text {sat }}\right)$. Como en el equilibrio de fases líquido-vapor del solvente puro se cumple la igualdad de las fugacidades, entonces se pueden igualar los coeficientes de fugacidad, así:

$$
\varphi_{j}^{L}\left(T, P_{j}^{\mathrm{sat}}\right)=\varphi_{j}^{V}\left(T, P_{j}^{\mathrm{sat}}\right)
$$

Esto permite definir una función objetivo $f(P)$,

$$
f(P)=\ln \left(\frac{\varphi_{j}^{L}}{\varphi_{j}^{V}}\right)
$$


que cumple la igualdad $f\left(P_{j}^{\mathrm{sat}}\right)=0$. De esta forma, a temperatura constante, $f$ mide qué tan cerca está $P$ a la presión de saturación del solvente puro. El valor de $P_{j}^{\text {sat }}$ se obtiene iterativamente buscando que $f=0$. El primer estimado de $P_{j}^{\text {sat }}$ proviene de la ecuación de Antoine (o de algún ajuste de datos similar), mientras que los estimados siguientes se obtienen con el método iterativo de la secante,

$$
P^{i+1}=P^{i}-\frac{f}{f^{\prime}}
$$

donde $f^{\prime}$ es una aproximación a la derivada $\operatorname{de} f$ (la secante) que depende de los resultados de las iteraciones anteriores:

$$
f^{\prime}=\frac{f^{i}-f^{i-1}}{P^{i}-P^{i-1}}
$$

donde los superíndices se refieren a las iteraciones (Llano, 1996). El criterio de detención de la búsqueda de $P_{j}^{\text {sat }}$ es la desigualdad $|f| \leq \varepsilon$, donde $\varepsilon$ es la tolerancia para la aproximación. Los coeficientes de fugacidad de líquido y vapor del solvente puro en la Ec. (37) se calculan con la ecuación de estado SRK para componente puro, así:

$$
\ln (\varphi)=\frac{A}{B} \ln \left(\frac{Z}{Z+B}\right)-\ln (Z-B)+Z-1
$$

en la que se reemplaza $Z$ del líquido o del vapor para calcular $\varphi_{j}^{L}$ y $\varphi_{j}^{V}$ según corresponda.

El término $\hat{\varphi}_{i}^{L}$ en la Ec. (11) se evalúa a $P=P_{j}^{\text {sat }}$ aplicando los límites $x_{j} \rightarrow 1$ y $x_{i} \rightarrow 0$ en la Ec. (32):

$\lim _{\substack{x_{i} \rightarrow 0 \\ x_{j} \rightarrow 1}} \ln \left(\hat{\varphi_{i}}\right)=\frac{B_{i}}{B_{j}}\left(Z_{j}-1\right)-\ln \left(Z_{j}-B_{j}\right)-\bar{\alpha}_{i} \ln \left(1+\frac{B_{j}}{Z_{j}}\right)$

con

$$
\bar{\alpha}_{i}=\frac{1}{\Lambda}\left[\ln \left(\gamma_{i}^{\infty}\right)+\ln \left(\frac{B_{j}}{B_{i}}\right)+\frac{B_{i}}{B_{j}}-1\right]+\frac{A_{i}}{B_{i}}
$$

Como $x_{i} \rightarrow 1, Z_{j}$ es el factor de compresibilidad del solvente $j$ líquido, y $\gamma_{i}^{\infty}$ es el coeficiente de actividad del soluto $i$ a dilución infinita.

\subsection{Implementación computacional}

La base de datos de parámetros del método PSRK está disponible como material complementario en el artículo de Horstmann et al. (2005) e incluye propiedades críticas, factores acéntricos, y parámetros de Mathias-Copeman para la ecuación de estado SRK; mientras que para el modelo UNIFAC incluye parámetros de volumen, superficie y de interacción en función de la temperatura para cada par de grupos de interacción, además de la información estructural de todos los componentes (es decir, cuántos grupos funcionales de cada tipo tiene cada molécula). Las tablas de parámetros se convirtieron en archivos de texto y luego en funciones computacionales que proporcionan los parámetros con base en el número de identificación de la sustancia. De esta forma es muy fácil añadir nuevos componentes a la base de datos, ya que las funciones computacionales son archivos de texto simple.

Buscando la mayor simplicidad posible y portabilidad se utilizaron los programas MATLAB y SCILAB como intérpretes de comandos, en lugar de lenguajes computacionales tradicionales como $\mathrm{C}++$ o FORTRAN. Aunque sus códigos no son exactamente iguales, las dos implementaciones (MATLAB y SCILAB) son numéricamente equivalentes, y los cálculos están distribuidos en las siguientes funciones (se omiten aquí las funciones auxiliares que proporcionan acceso a la base de datos de los componentes):

EOS PSRK: a partir de los parámetros $T, P, y$ (composición molar), y con base en las identidades de los compuestos, obtiene los factores de compresibilidad $Z$ con la Ec. (22) (uno por cada fase en coexistencia a $T$ y $P$ ), y los coeficientes de fugacidad parciales $\hat{\varphi}_{i}$ para todos los componentes $i$ en todas las fases. Para prevenir la aparición de los problemas numéricos descritos por Zhi \& Lee (2002), se usan los métodos numéricos de solución de raíces de polinomios que vienen incluidos en MATLAB y SCILAB en 
lugar del método analítico de Cardano y Vieta para resolver la ecuación cúbica.

bublp_PSRK: con la temperatura y la composición del líquido (y un estimado inicial de la presión), calcula la presión del sistema, la composición del vapor en el equilibrio y los factores de compresibilidad de ambas fases (algoritmo Burbuja- $P$ ). Utiliza como función objetivo $f(P)=\sum_{i} y_{i}-1$ para obtener $P$ iterativamente. Las relaciones entre las fracciones molares del componente $i$ en el líquido y en el vapor se obtienen de la expresión

$$
K_{i}=y_{i} / x_{i}=\hat{\varphi}_{i}^{L} / \hat{\varphi}_{i}^{V}
$$

psat_PSRK: para una sustancia pura obtiene la presión de saturación en función de $T$. Usa la Ec. (37) y requiere un estimado inicial de la presión de saturación.

KH_PSRK : calcula la constante de la ley de Henry en función de la temperatura usando las Ecs. (11) y (42). Esta función requiere un estimado inicial de la presión de saturación. Si el solvente es una sustancia pura, $P_{j}^{\text {sat }}$ se obtiene con psat_PSRK; si es una mezcla, emplea el algoritmo Burbuja- $P$ en la función bublp_PSRK.

Para el lector interesado, los autores tienen disponibles las funciones anteriores, junto con las instrucciones de uso.

\section{Resultados y discusión}

La implementación computacional construida en el presente trabajo se puso a prueba comparando los resultados de la Ec. (11) con un conjunto reciente de medidas experimentales de $H_{i, j}$ de gases disueltos en 10 tipos de alcoholes, con un total de 48 pares soluto-solvente y 258 mediciones a varias temperaturas (ver Tabla 1), y también con las medidas de $H_{i, j}$ del 1-buteno disuelto en las mezclas metanol-benceno y metanol-ciclohexano, reportadas por Miyano et al. (2003a). Los resultados se graficaron en función de la temperatura para cada solvente (Figuras 1-12).
La concordancia entre el método PSRK y las mediciones experimentales se cuantificó con el porcentaje de error, definido como

$$
e_{H}=100\left[\frac{\left(H_{i, j}\right)^{\mathrm{calc}}-\left(H_{i, j}\right)^{\exp }}{\left(H_{i, j}\right)^{\exp }}\right]
$$

donde los superíndices calc y exp se refieren al valor calculado con el método PSRK y al valor experimental, respectivamente. Los valores de $e_{H}$ para gases disueltos en solventes puros se muestran en la Tabla 1, promediados por soluto y por solvente. Los resultados fueron similares a los del estudio de Apostolou et. al.(1995). Para la gran mayoría de los pares soluto-solvente los valores de $e_{H}$ están por debajo del $25 \%$, pero hay sistemas en los que no se cumple esta tendencia: aquellos con 2-propen-1ol como solvente (todos los solutos) y los pares 1,3 butadieno-1-pentanol y 1,3 butadieno-1-propanol. Esto sugiere que las predicciones del método PSRK para $H_{i, j}$ tienden a alejarse de los datos experimentales cuando el soluto o el solvente es una sustancia orgánica con dobles enlaces. Solucionar este problema podría requerir una reparametrización de los grupos funcionales de UNIFAC-PSRK, algo poco probable en el estado actual de desarrollo del modelo.

En todos los pares soluto-solvente puro el método PSRK reprodujo el incremento de $H_{i, j}$ con la temperatura, debido a que la fugacidad del gas aumenta y su solubilidad disminuye, un comportamiento que se explica en términos termodinámicos con la siguiente expresión (válida a bajas concentraciones):

$$
\left(\frac{\partial \ln x_{i}}{\partial T}\right)_{P}=-\frac{\left(\hat{H}_{i}^{G}-\bar{H}_{i}^{L}\right)}{R T^{2}}
$$

Dado que la entalpía del gas es usualmente mayor que la del líquido, el lado derecho es negativo y la solubilidad disminuye con la temperatura (Prausnitz et al., 2000). Por eso resulta muy interesante la reproducción del máximo en $H_{i, j}$ con el metanol como solvente (Figura 1). 
Tabla 1. Resultados obtenidos incluyendo los errores promedio y el rango de temperatura.

\begin{tabular}{|c|c|c|c|c|}
\hline Solvente & Soluto & $\begin{array}{c}e_{H} \\
\text { (promedio) } \\
\end{array}$ & Rango de $T(\mathrm{~K})$ & $\begin{array}{l}\text { Fuente de datos } \\
\text { experimentales }\end{array}$ \\
\hline \multirow[t]{5}{*}{ Metanol } & 1-buteno & 5.8 & $255.06-320.09$ & \multirow{4}{*}{$\begin{array}{l}\text { Miyano et al. (2006a) } \\
\text { Miyano et al. (2003b) }\end{array}$} \\
\hline & 2-metilpropano & 8.0 & $374.00-513.38$ & \\
\hline & butano & 15.8 & $255.05-513.38$ & \\
\hline & propano & 4.8 & $374.00-513.38$ & \\
\hline & GLOBAL & 10.4 & & \\
\hline \multirow[t]{6}{*}{ 1-propanol } & 1.3-butadieno & 58.8 & $259.99-339.99$ & \multirow{5}{*}{ Miyano (2004d) } \\
\hline & 1-buteno & 7.6 & $260.03-340.00$ & \\
\hline & butano & 23.1 & $260.05-340.04$ & \\
\hline & propano & 23.2 & $259.98-339.95$ & \\
\hline & trans-2-buteno & 5.8 & $260.02-340.06$ & \\
\hline & GLOBAL & 23.7 & & \\
\hline \multirow[t]{5}{*}{ 2-propanol } & 1-buteno & 4.4 & $249.97-330.00$ & \multirow{4}{*}{ Miyano (2004b) } \\
\hline & butano & 26.3 & $249.97-330.00$ & \\
\hline & propano & 25.7 & $249.97-330.00$ & \\
\hline & trans-2-buteno & 10.5 & $249.97-330.00$ & \\
\hline & GLOBAL & 16.7 & & \\
\hline \multirow[t]{10}{*}{ 2-propen-1-ol } & cloroetano & 41.5 & $249.94-329.99$ & \multirow{9}{*}{ Miyano (2005a) } \\
\hline & 1,3-butadieno & 8.8 & $250.00-329.99$ & \\
\hline & 1-buteno & 36.4 & $249.94-329.99$ & \\
\hline & 2-metilpropano & 53.1 & $249.94-329.99$ & \\
\hline & butano & 52.4 & $249.94-329.99$ & \\
\hline & cis-2-buteno & 34.7 & $249.94-329.99$ & \\
\hline & dimetileter & 33.3 & $249.94-329.99$ & \\
\hline & propano & 49.9 & $249.94-329.99$ & \\
\hline & trans-2-buteno & 40.0 & $250.00-329.99$ & \\
\hline & GLOBAL & 38.9 & & \\
\hline \multirow[t]{5}{*}{ 1-butanol } & 1-buteno & 6.1 & $250.01-330.00$ & \multirow{4}{*}{ Miyano (2004a) } \\
\hline & Butano & 20.4 & $250.01-330.00$ & \\
\hline & Propano & 18.8 & $250.01-330.00$ & \\
\hline & Trans-2-buteno & 5.8 & $250.01-330.00$ & \\
\hline & GLOBAL & 12.8 & & \\
\hline \multirow[t]{5}{*}{ 2-butanol } & 1-buteno & 2.9 & $249.97-330.00$ & \multirow{4}{*}{ Miyano (2004a) } \\
\hline & butano & 22.8 & $249.96-330.00$ & \\
\hline & propano & 20.7 & $249.97-330.00$ & \\
\hline & trans-2-buteno & 11.7 & $249.97-330.00$ & \\
\hline & GLOBAL & 14.5 & & \\
\hline \multirow[t]{6}{*}{ 1-pentanol } & 1.3-butadieno & 46.0 & $249.97-330.00$ & \multirow{5}{*}{ Miyano (2005c) } \\
\hline & 1-buteno & 7.2 & $249.96-330.00$ & \\
\hline & butano & 15.4 & $249.98-330.00$ & \\
\hline & propano & 14.7 & $249.96-330.00$ & \\
\hline & trans-2-buteno & 3.7 & $249.96-330.00$ & \\
\hline & GLOBAL & 17.4 & & \\
\hline \multirow[t]{6}{*}{ 2-pentanol } & 1.3-butadieno & 26.3 & $249.94-330.00$ & \multirow{5}{*}{ Miyano (2005c) } \\
\hline & 1-buteno & 1.6 & $249.94-330.00$ & \\
\hline & butano & 18.2 & $249.93-330.00$ & \\
\hline & propano & 18.8 & $249.93-330.00$ & \\
\hline & trans-2-buteno & 7.1 & $249.94-330.00$ & \\
\hline & GLOBAL & 14.4 & & \\
\hline \multirow[t]{5}{*}{ 2-metil-1-butanol } & 1-buteno & 3.2 & $250.05-329.97$ & \\
\hline & butano & 17.1 & $250.05-329.97$ & Mivano (2005b) \\
\hline & propano & 18.1 & $250.05-329.97$ & 1911у वा10 (2000) \\
\hline & trans-2-buteno & 8.4 & $250.05-329.97$ & \\
\hline & GLOBAL & 11.7 & & \\
\hline 3-metil-1-butanol & 1-buteno & 5.7 & $249.92-330.00$ & \\
\hline & butano & 15.7 & $249.92-330.00$ & Mivano $(2005 \mathrm{~h})$ \\
\hline & propano & 13.9 & $249.92-330.00$ & 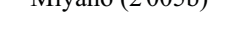 \\
\hline & trans-2-buteno & 5.3 & $249.92-330.00$ & \\
\hline & GLOBAL & 10.1 & & \\
\hline
\end{tabular}




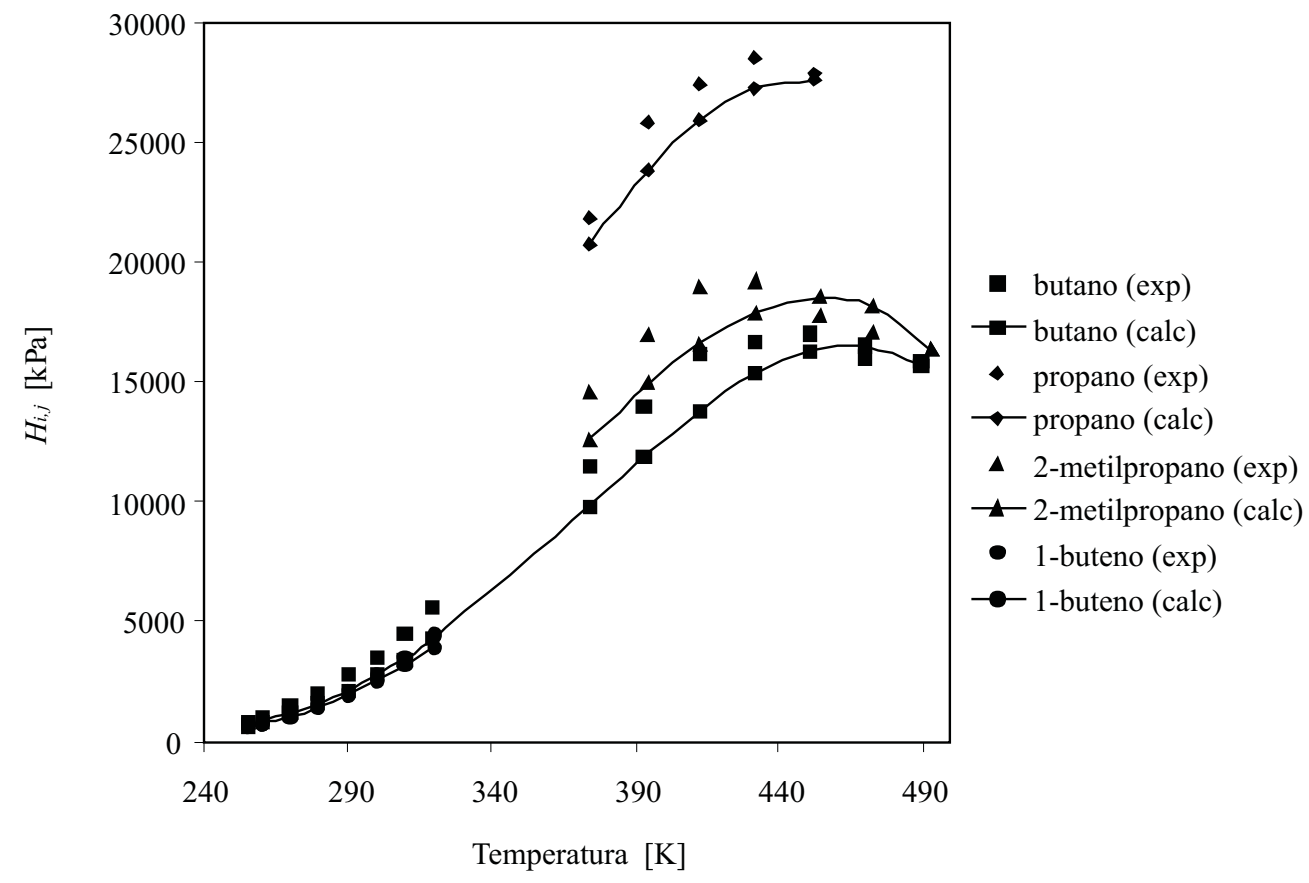

Figura 1. Constante de la ley de Henry en función de la temperatura para varios gases disueltos en metanol. (Las fuentes de datos experimentales están listadas en la Tabla 1).

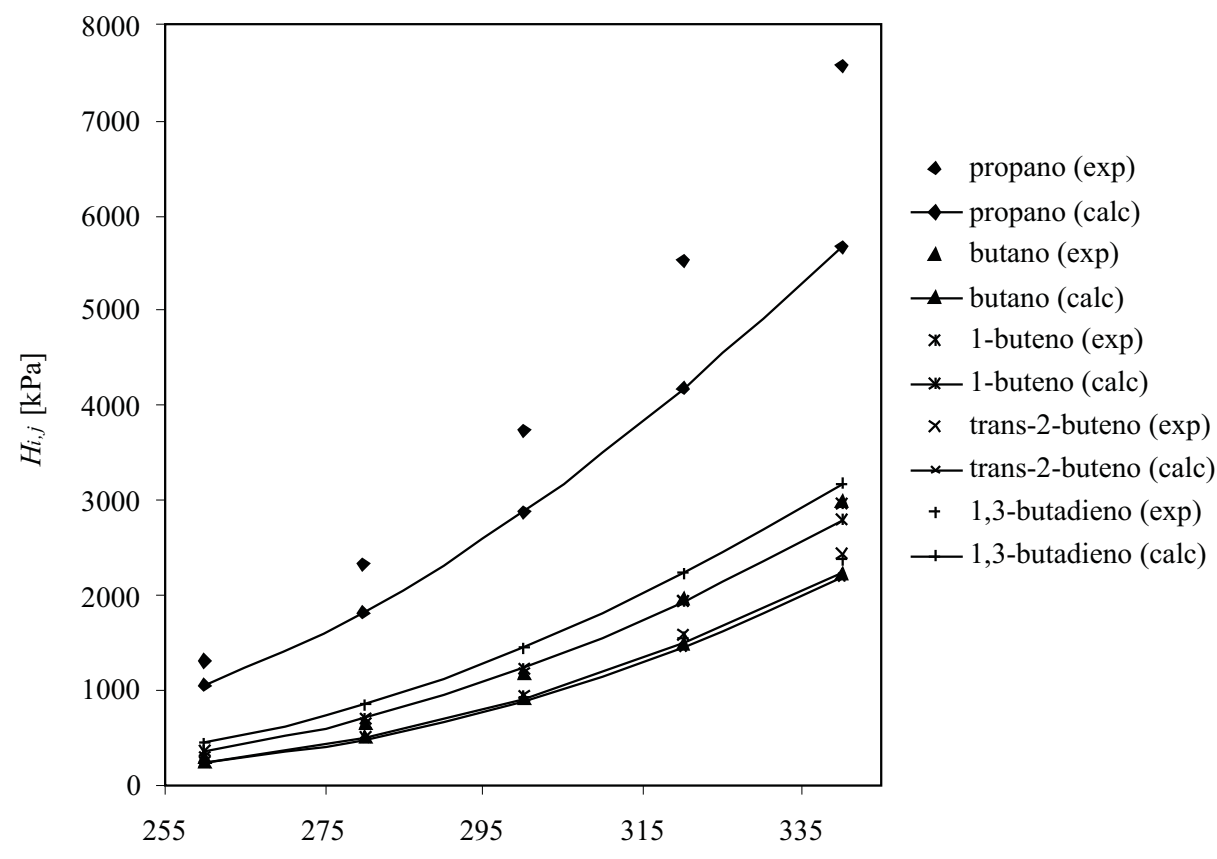

Temperatura $[\mathrm{K}]$

Figura 2. Constante de la ley de Henry en función de la temperatura para varios gases disueltos en 1-propanol. (Las fuentes de datos experimentales están listadas en la Tabla 1). 


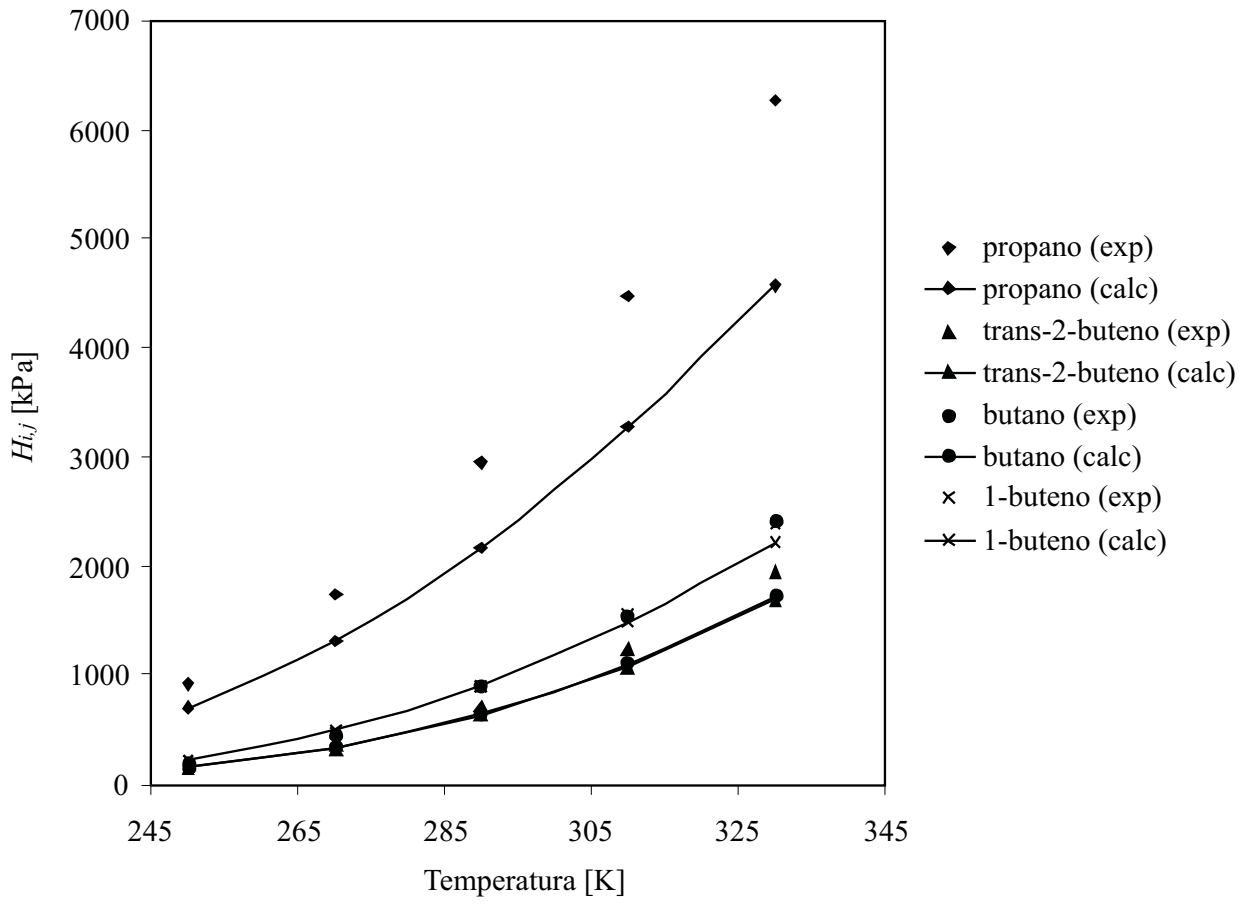

Figura 3. Constante de la ley de Henry en función de la temperatura para varios gases disueltos en 2-propanol. (Las fuentes de datos experimentales están listadas en la Tabla 1).

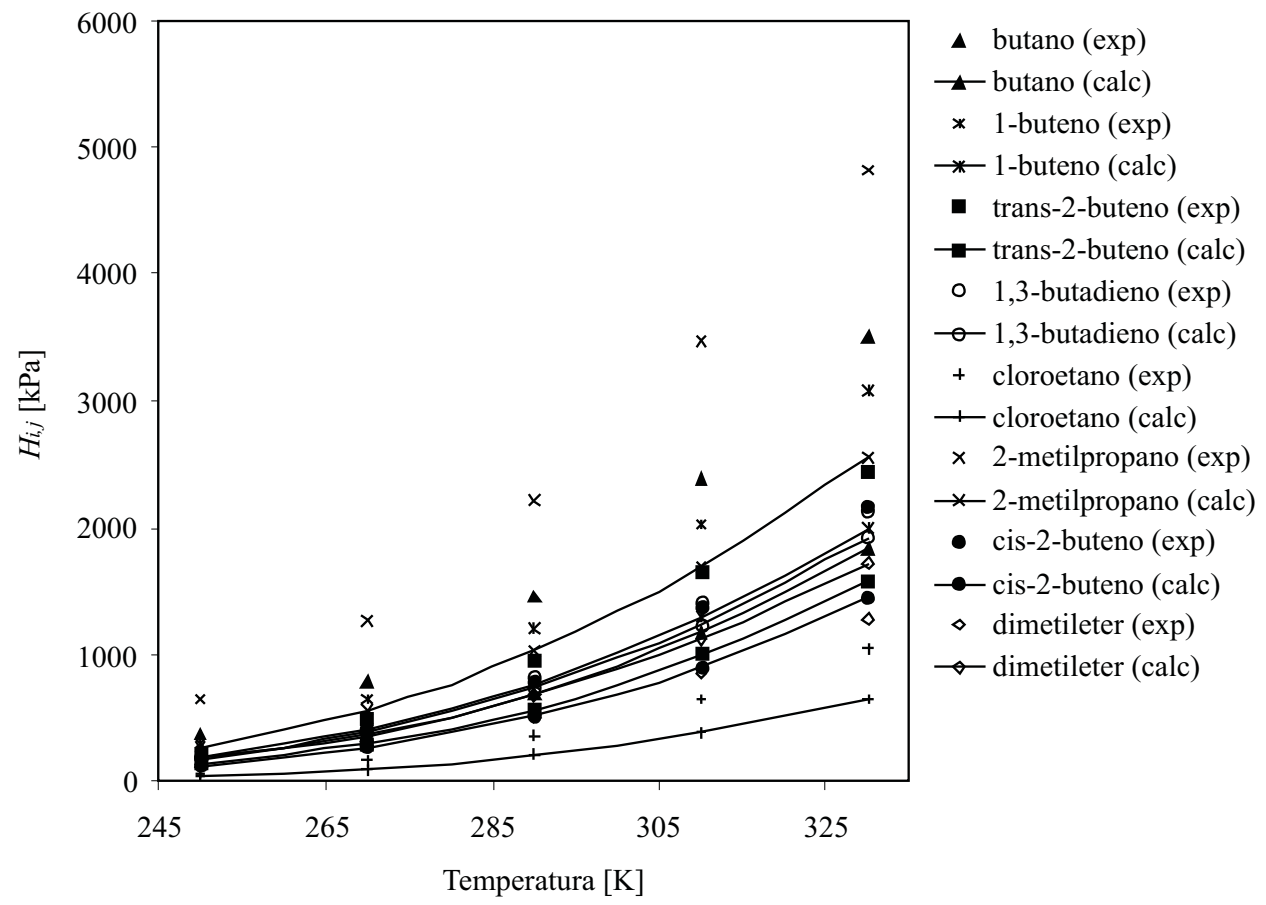

Figura 4. Constante de la ley de Henry en función de la temperatura para varios gases disueltos en 2-propen-1-ol. (Las fuentes de datos experimentales están listadas en la Tabla 1). 


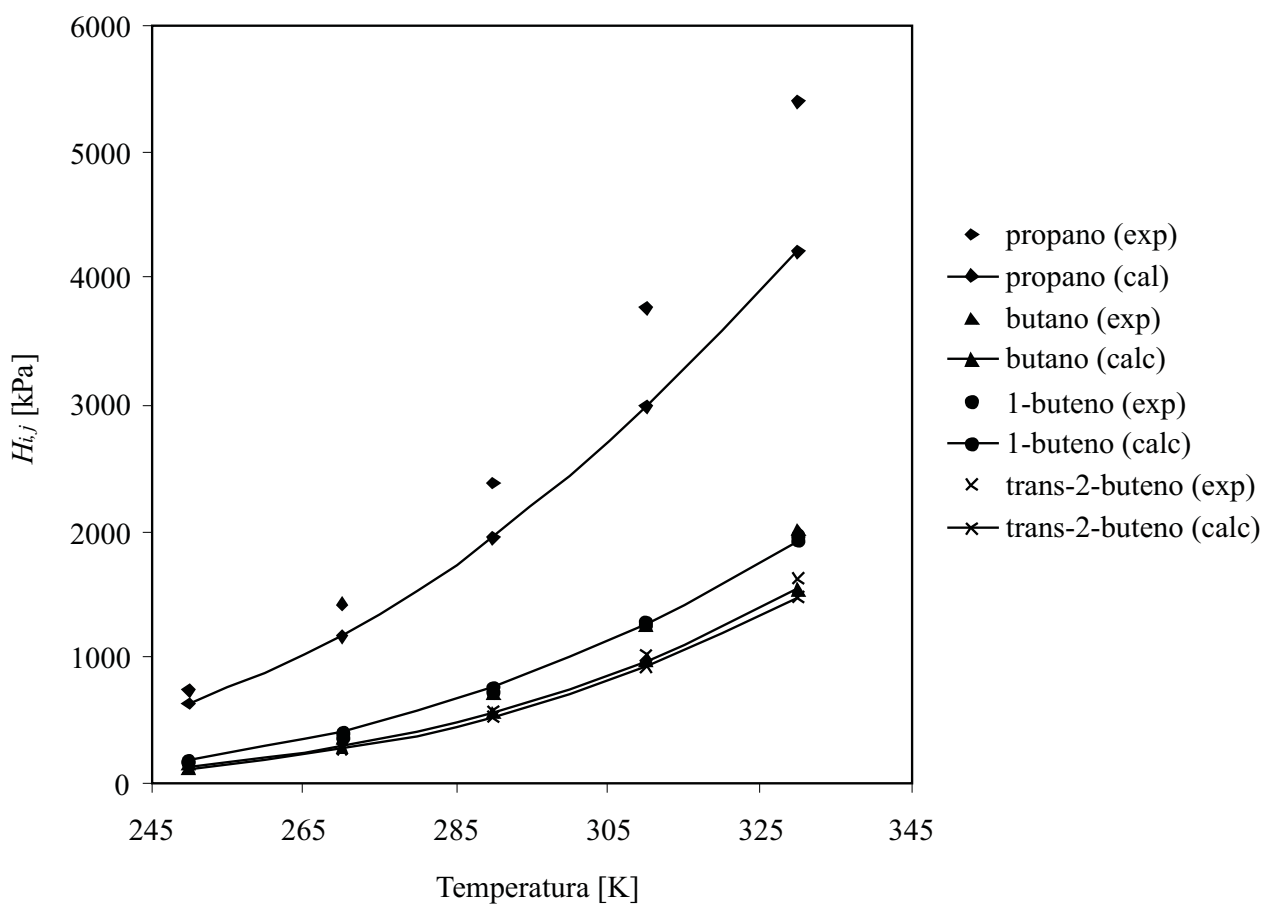

Figura 5. Constante de la ley de Henry en función de la temperatura para varios gases disueltos en 1-butanol. (Las fuentes de datos experimentales están listadas en la Tabla 1).

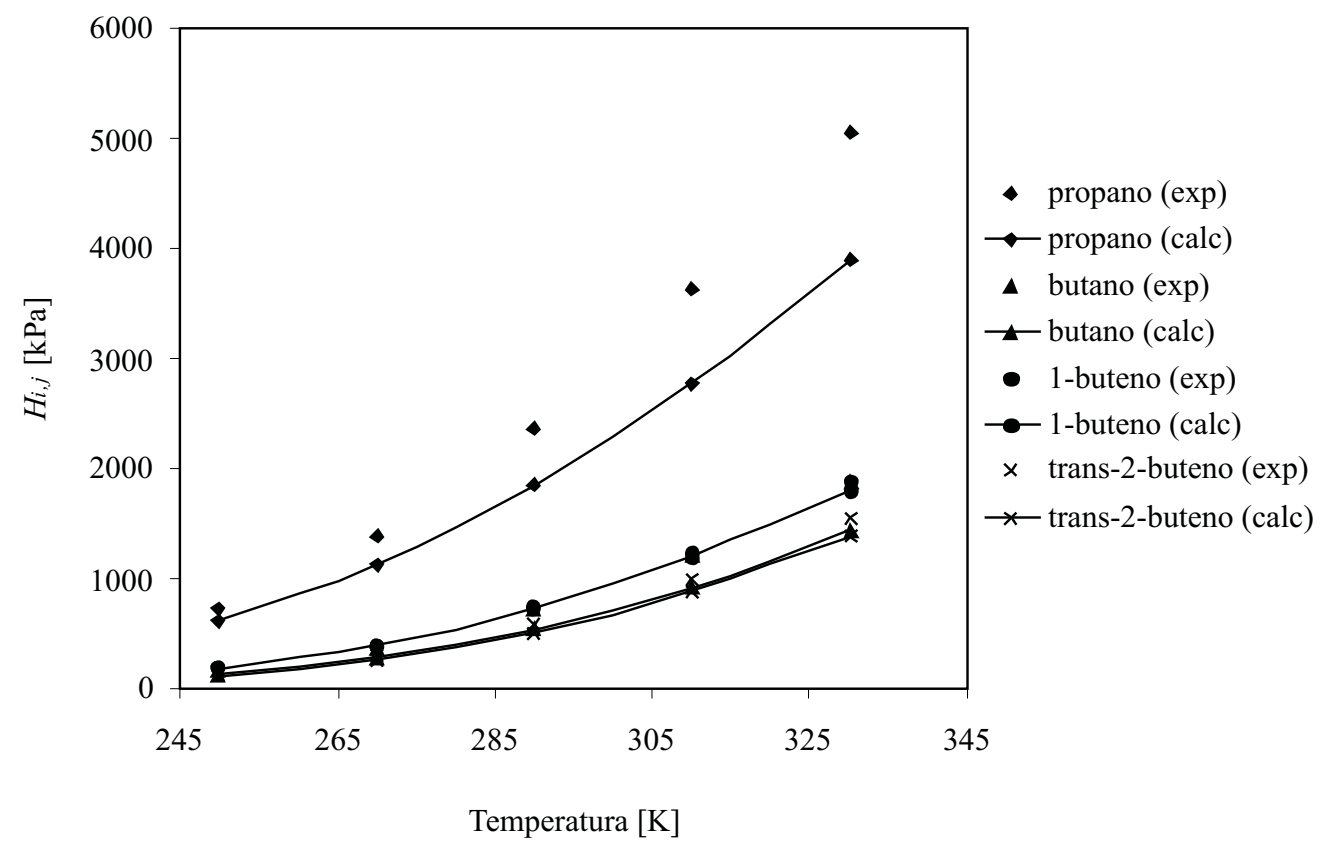

Figura 6. Constante de la ley de Henry en función de la temperatura para varios gases disueltos en 2-butanol. (Las fuentes de datos experimentales están listadas en la Tabla 1). 


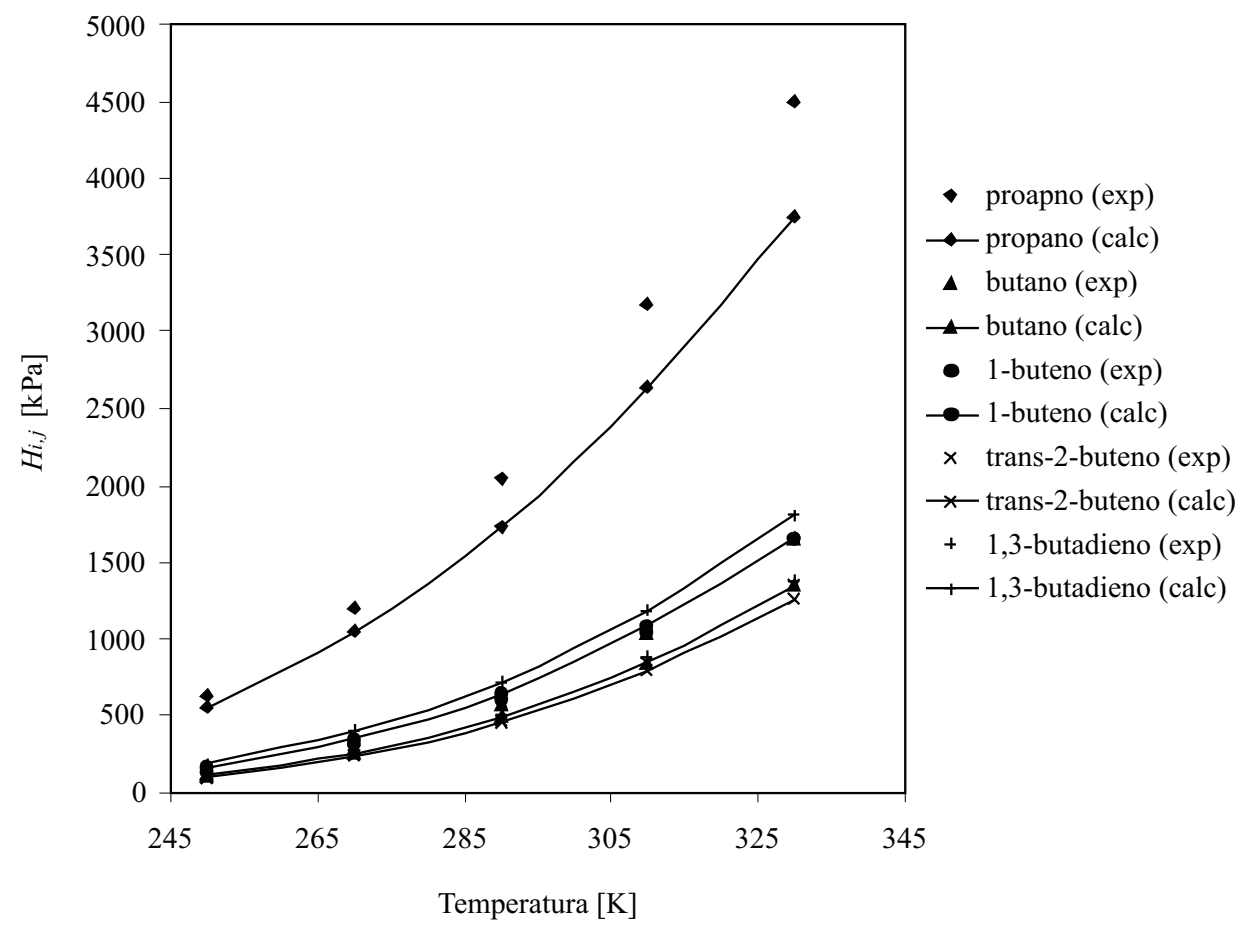

Figura 7. Constante de la ley de Henry en función de la temperatura para varios gases disueltos en 1-pentanol. (Las fuentes de datos experimentales están listadas en la Tabla 1).

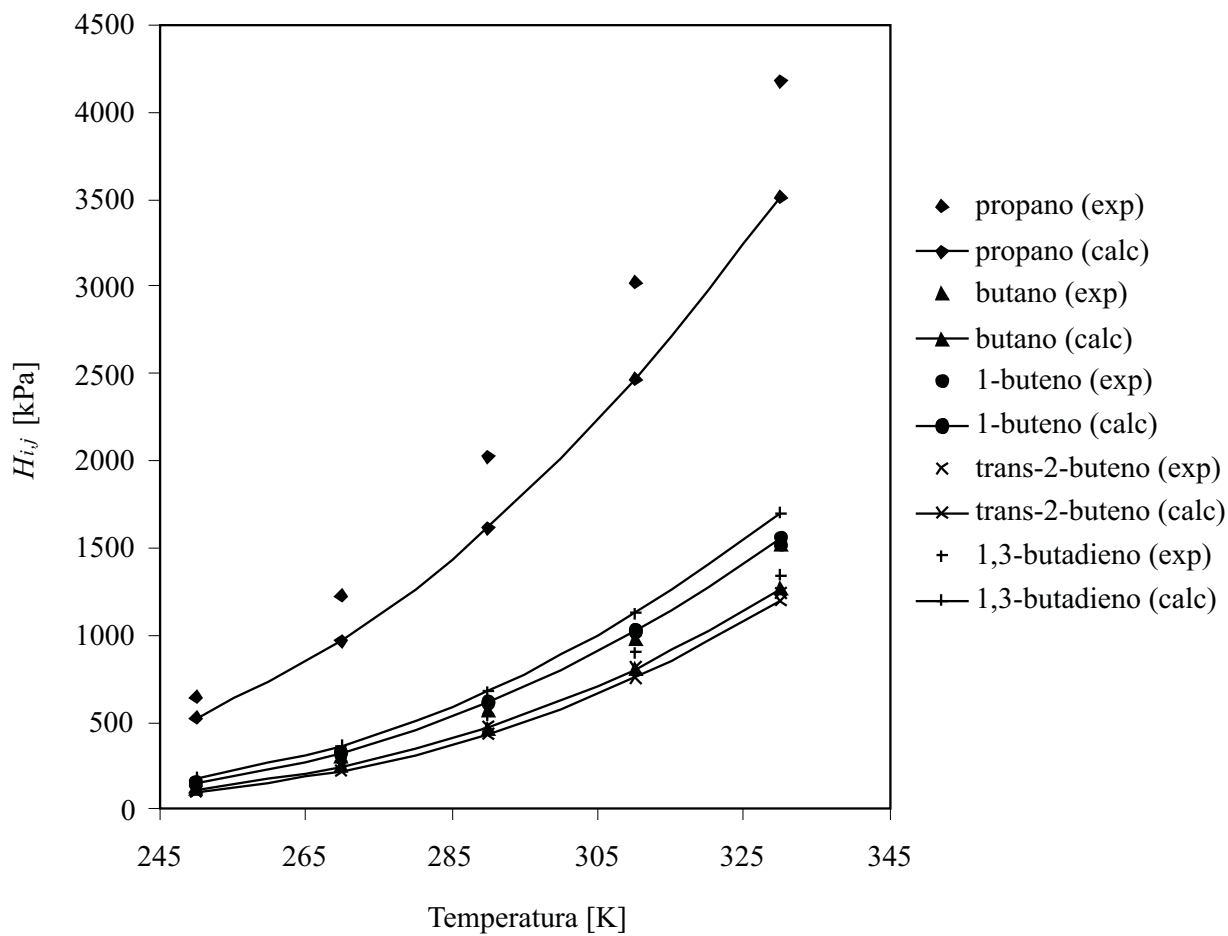

Figura 8. Constante de la ley de Henry en función de la temperatura para varios gases disueltos en 2-pentanol. (Las fuentes de datos experimentales están listadas en la Tabla 1). 


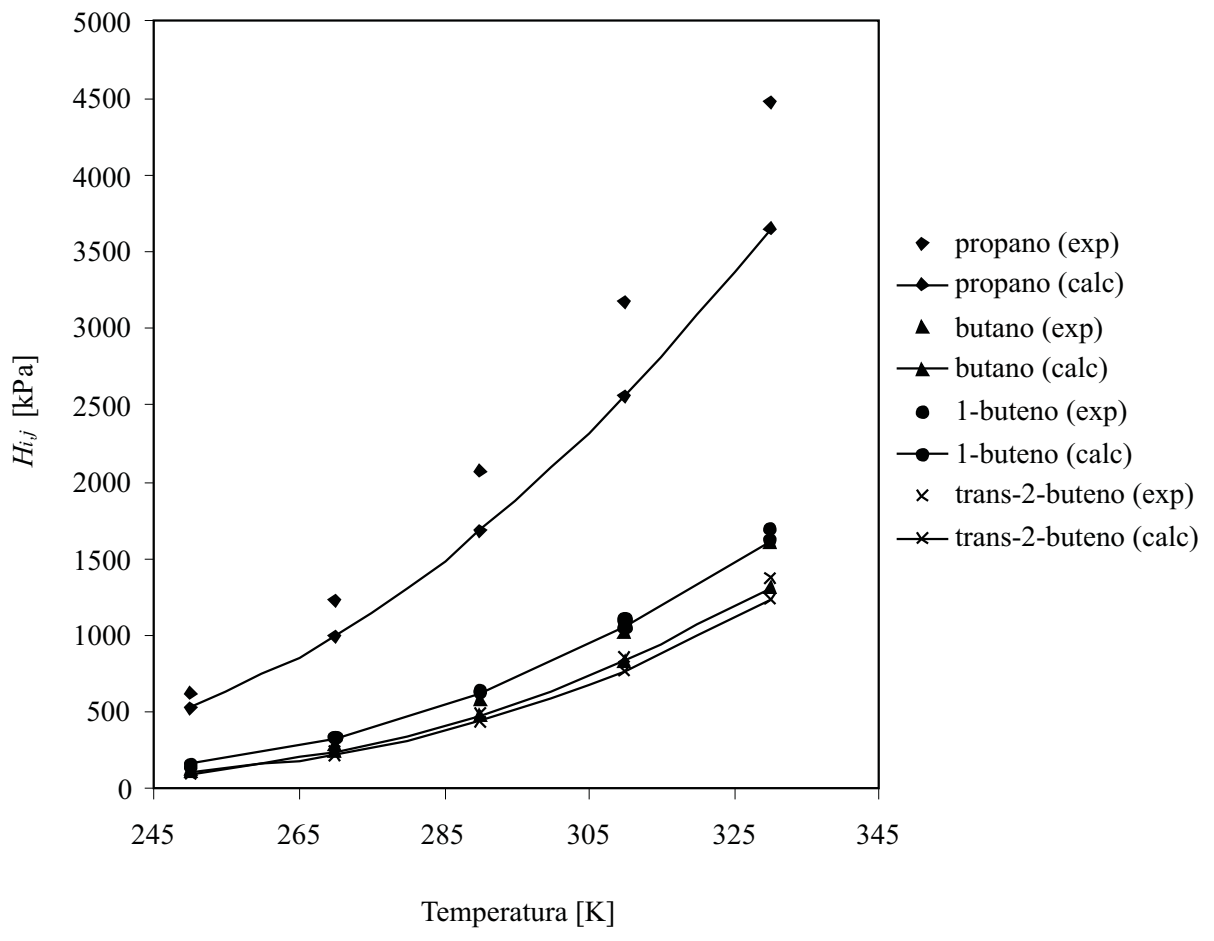

Figura 9. Constante de la ley de Henry en función de la temperatura para varios gases disueltos en 2-metil-1-butanol. (Las fuentes de datos experimentales están listadas en la Tabla 1).

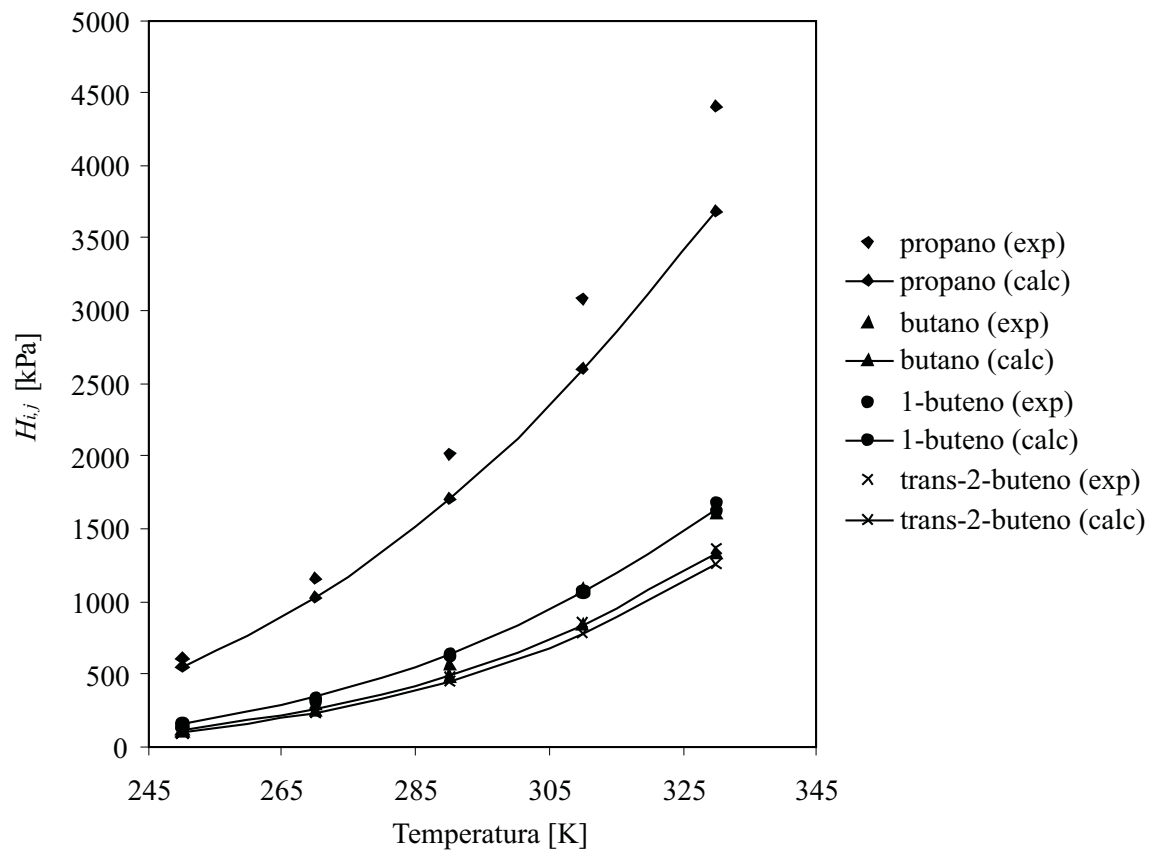

Figura 10. Constante de la ley de Henry en función de la temperatura para varios gases disueltos en 3-metil-1-butanol. (Las fuentes de datos experimentales están listadas en la Tabla 1). 


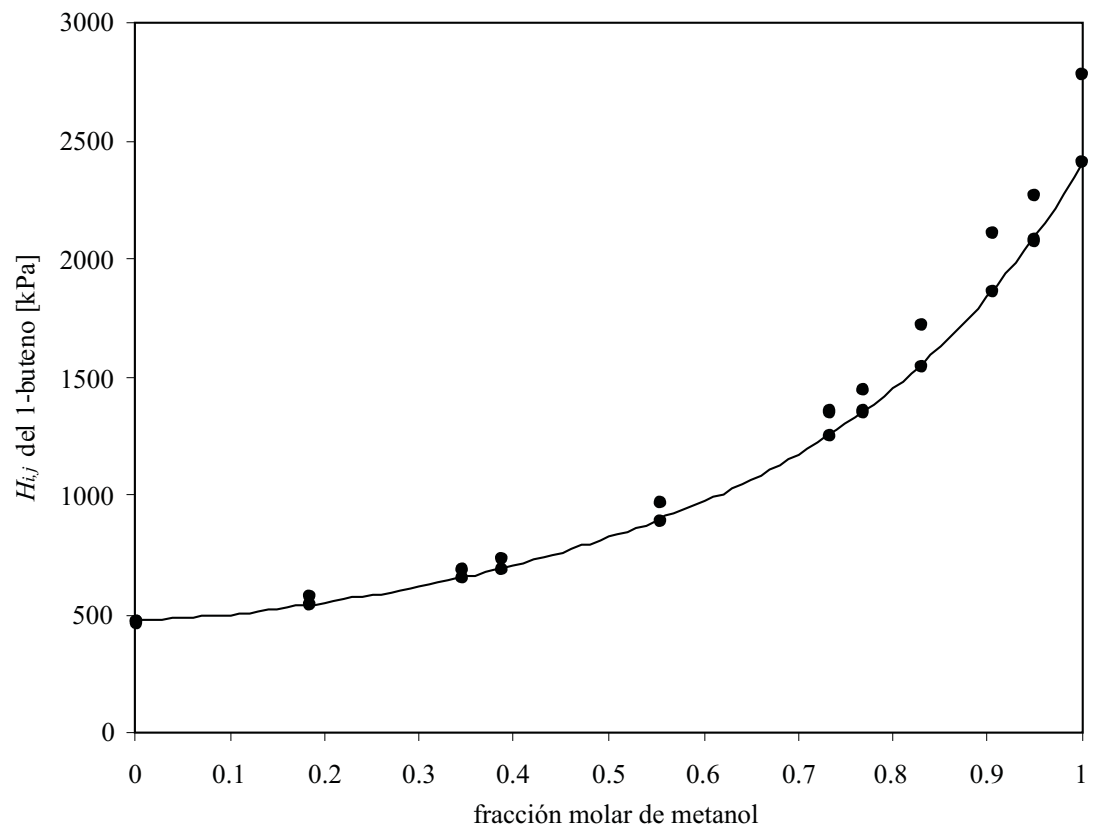

Figura 11. Constante de la ley de Henry del 1-buteno disuelto en una mezcla metanol-benceno a $298.15 \mathrm{~K}$ en función de la fracción molar de metanol. Círculos: resultados experimentales, círculos unidos por una linea: valores calculados. [Fuente de datos experimentales: Miyano et al. (2003a)].

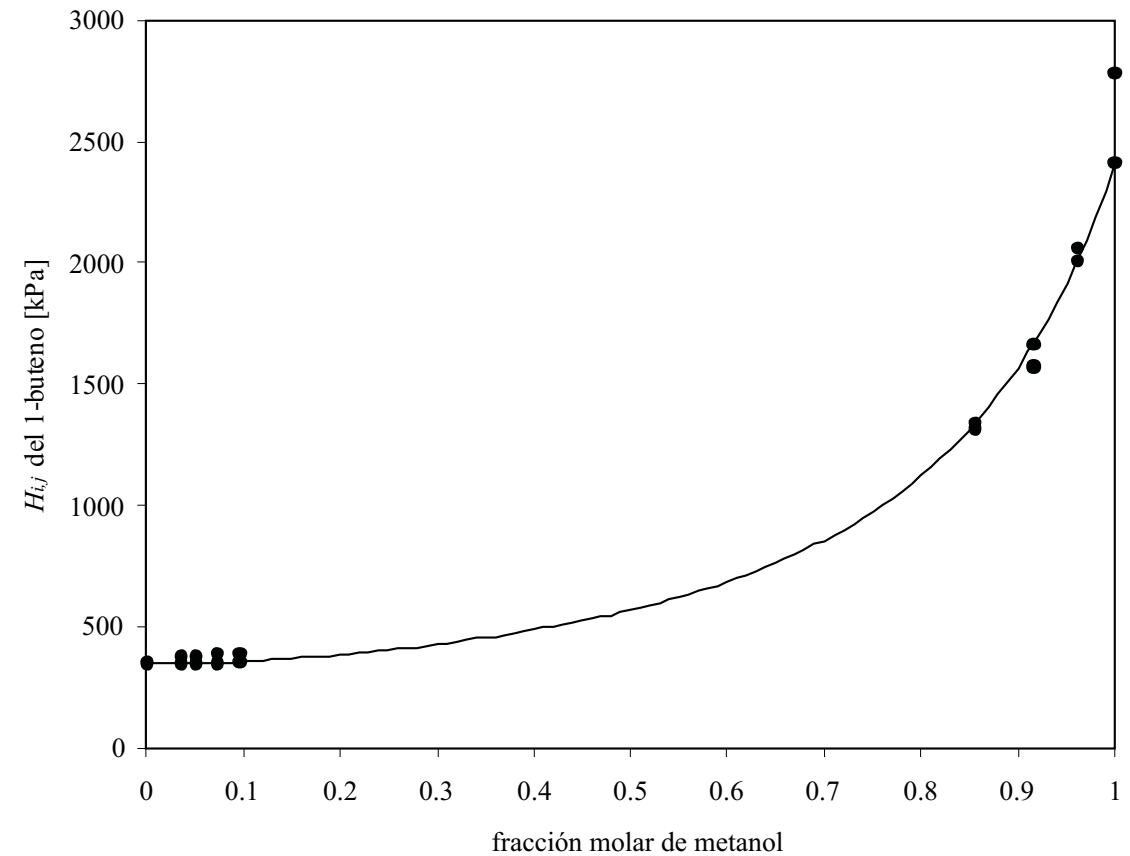

Figura 12. Constante de la ley de Henry del 1-buteno disuelto en una mezcla metanol-ciclohexano a $298.15 \mathrm{~K}$ en función de la fracción molar de metanol. Círculos: resultados experimentales, círculos unidos por una línea: valores calculados. [Fuente de datos experimentales: Miyano et al. (2003a)]. 
Este comportamiento peculiar se explica por cambios en las interacciones soluto-solvente inducidas por la temperatura que pueden cambiar el signo de la cantidad $\hat{H}_{i}^{G}-\vec{H}_{i}^{L}$ de positivo a negativo, de forma que un incremento de la temperatura implica una disminución de $H_{i, j}$ (o un aumento en la solubilidad), de acuerdo con Prausnitz et al. (2000).

En términos moleculares, se puede explicar también que las fuerzas de los enlaces de hidrógeno entre las moléculas del solvente (metanol) alcanzan un máximo, disminuyendo la solubilidad al impedir que las moléculas de soluto ocupen los intersticios entre las moléculas de solvente. Cuando la temperatura aumenta, las fuerzas de los enlaces de hidrógeno se debilitan, permitiendo que ingresen más moléculas del soluto gaseoso (Mullins et al., 1998). Este comportamiento ya había sido predicho por el método PSRK para el sistema hidrógeno-agua (Horstmann et al., 2000).

Para el 1-buteno disuelto en las mezclas metanolbenceno y metanol-ciclohexano se obtuvo una reproducción bastante buena de los resultados experimentales (Figuras 11 y 12). El valor promedio de $e_{H}$ fue de 7.8 con la mezcla metanolbenceno como solvente y de 6.7 con la mezcla metanol-ciclohexano. Estos resultados se comparan bastante bien con los obtenidos con solventes puros.

\section{Conclusiones}

El conjunto de funciones computacionales desarrolladas en este trabajo y la base de datos Dortmund Data Bank permiten aplicar el método PSRK a problemas de equilibrio de fases líquidovapor, incluyendo el cálculo de la constante de la ley de Henry y eliminando la necesidad de que el usuario busque parámetros y haga un largo trabajo de programación. Al usar las funciones computacionales para reproducir valores de $H_{i, j}$ recientemente publicados, se ha reforzado la conclusión de trabajos previos, de que el método PSRK es válido para predecir valores de constantes de la ley de Henry en sistemas orgánicos, y que también tiene la capacidad de reproducir el comportamiento de $H_{i, j}$ con la temperatura. Sin embargo, aunque son útiles, las predicciones de $H_{i, j}$ hechas con el método PSRK muestran un alejamiento con respecto a los datos experimentales que varía mucho de un sistema a otro, de forma que estas predicciones no pueden tomarse como un reemplazo de las mediciones experimentales, sino como una herramienta útil de aproximación en aplicaciones didácticas y cálculos de ingeniería.

\section{Referencias bibliográficas}

Apostolou, D. A., Kalospiros, N. S., \& Tassios, D. P. (1995). Prediction of gas solubilities using the LCVM equation of state/excess Gibbs free energy model. Industrial \& Engineering Chemistry Research 34 (3), 984-957.

Bader, M. S. H., \& Gasem, K. A. M. (1996). Prediction of phase behavior, Henry's constants, and infinite dilution partial molar volumes for carbon dioxide and carbon monoxide in n-paraffins. Journal of Supercritical Fluids 9 (4), 244-262.

Boukouvalas, C., Spiliotis, N., Coutsikos, P., Tzouvaras, N., \& Tassios, D. (1994). Prediction of vapor-liquid equilibrium with the LCVM model: a linear combination of the Vidal and Michelsen mixing rules coupled with the original UNIFAC and the t-mPR equation of state. Fluid Phase Equilibria 92, 75-106.

Chen, J., Fischer, K., \& Gmehling, J. (2002). Modification of PSRK mixing rules and results for vapor-liquid equilibria, enthalpy of mixing and activity coefficients at infinite dilution. Fluid Phase Equilibria 200 (2), 411-429.

Cichowski, E. C., Schmidt, T. R., \& Errington, J. R. (2005). Determination of Henry's law constants through transition matrix Monte Carlo simulation. Fluid Phase Equilibria 236 (1-2), $58-65$.

Dahl, S., \& Michelsen, M. L. (1990). Highpressure vapor-liquid equilibrium with a UNIFAC-based equation of state. AIChE Journal 36(12), 1829-1836. 
Dahl, S., Fredenslund, A., \& Rasmussen, P. (1991). The MHV2 model: A UNIFAC-based equation of state model for prediction of gas solubility and vapor-liquid equilibria at low and high pressures. Industrial \& Engineering Chemistry Research 30 (8), 1936-1945.

Fischer, K., \& Gmehling, J. (1996). Further development, status and results of the PSRK method for the prediction of vapor-liquid equilibria and gas solubilities. Fluid Phase Equilibria 121 (1-2), 185-206.

Fredenslund, A., Jones, R. L., \& Prausnitz, J. M. (1975). Group contribution estimation of activity coefficients in nonideal liquid mixtures. AIChE Journal 21 (6), 1086-1099.

Fredenslund, A., Gmehling, J., Michelsen, M. L., Rasmussen, P., \& Prausnitz, J. M. (1977a). Computerized design of multicomponent distillation columns using the UNIFAC group contribution method for calculation of activity coefficients. Industrial \& Engineering Chemistry Process Design and Development 16 (4), 450-462.

Fredenslund, A., Gmehling, J., \& Rasmussen, P. (1977b). Vapor-liquid equilibria using UNIFAC. Amsterdam:Elsevier.

Gmehling, J., Li, J., \& Fischer, K. (1997). Further development of the PSRK model for the prediction of gas solubilities and vapor-liquid equilibria at low and high pressures II. Fluid Phase Equilibria 141 (1-2), 113-127.

Holderbaum, T., \& Gmehling, J. (1991). PSRK: a group contribution equation of state based on UNIFAC. Fluid Phase Equilibria 70 (2-3), 251-265.

Horstmann, S., Fischer, K., \& Gmehling, J. (2000). PSRK group contribution equation of state: revision and extension III. Fluid Phase Equilibria 167 (2), 173-186.
Horstmann, S., Jabloniec, A., Krafczyk, J., Fischer, K., \& Gmehling, J. (2005). PSRK group contribution equation of state: comprehensive revision and extension IV, including critical constants and $\alpha$-function parameters for 1000 components. Fluid Phase Equilibria 227(2), $157-164$.

Huron, M. J., \& Vidal, J. (1979). New mixing rules in simple equations of state for representing vapour-liquid equilibria of strongly non-ideal mixtures. Fluid Phase Equilibria 3 (4), 255-271.

IFPS (Industrial Fluid Properties Simulation Collective). (2008). Second industrial fluid properties simulation challenge: problem 2 Henry's law constant.

http://fluidproperties.org/problems/problem2.htm

Jantunen, L. M., \& Bidleman, T. F. (2006). Henry's law constants for hexachlorobenzene, $p, p^{\prime}-D D E$ and components of technical chlordane and estimates of gas exchange for Lake Ontario. Chemosphere 62(10), 1689-1696.

Leroi, J. C., Masson, J. C., Renon, H., Fabries, J. F., \& Sannier, H. (1977). Accurate measurement of activity coefficients at infinite dilution by inert gas stripping and gas chromatography. Industrial \& Engineering Chemistry Process Design and Development 16(1), 139-144.

Li, J., Fischer, K., \& Gmehling, J. (1998). Prediction of vapor-liquid equilibria for asymmetric systems at low and high pressures with the PSRK model. Fluid Phase Equilibria 143 (1-2), 71-82.

Llano, M. A. (1996). Conferencias de clase del curso de termodinámica química. Departamento de Procesos Químicos y Biológicos, Facultad de Ingeniería, Universidad del Valle, Cali, Colombia.

Michelsen, M. L. (1990). A modified Huron-Vidal mixing rule for cubic equations of state. Fluid Phase Equiilbria 60 (1-2), 213-219. 
Miyano, Y., \& Nakanishi, K. (2003a). Solubilities of 1-butene in (methanol+benzene) and (methanol + cyclohexane) at $\mathrm{T}=298.15 \mathrm{~K}$ and $\mathrm{p}=40 \mathrm{kPa}$ to $102 \mathrm{kPa}$. Journal of Chemical Thermodynamics 35 (3), 519-528.

Miyano, Y., Nakanishi, K., \& Fukuchi, K. (2003b). Henry's constants of butane, isobutane, 1-butene and isobutene in methanol at 255-320 K. Fluid Phase Equilibria 208 (1-2), 223-238.

Miyano, Y. (2004a). Henry's constants and infinite dilution activity coefficients of propane, propene, butane, isobutane, 1-butene, isobutene, trans-2-butene, and 1,3-butadiene in 1-butanol and 2-butanol from (250 to 330) K. Journal of Chemical \& Engineering Data 49 (5), 1285-1289.

Miyano, Y. (2004b). Henry's constants and infinite dilution activity coefficients of propane, propene, butane, isobutane, 1-butene, isobutene, trans-2-butene, and 1,3-butadiene in 2-propanol at 250-330 K. Journal of Chemical \& Engineering Data 49(2), 368-371.

Miyano, Y. (2004c). Henry's constants and infinite dilution activity coefficients of propane, propene, butane, isobutane, 1-butene, isobutene, trans-2-butene, and 1,3-butadiene in isobutanol and tert-butanol. Journal of Chemical Thermodynamics 36(10), 865-869.

Miyano, Y. (2004d). Henry's constants and infinite dilution activity coefficients of propane, propene, butane, isobutene, 1-butene, isobutane, trans-2-butene and 1,3-butadiene in 1-propanol at $\mathrm{T}=(260$ to 340$) \mathrm{K}$. Journal of Chemical Thermodynamics 36(2), 101-106.

Miyano, Y. (2005a). Henry's law constants and infinite dilution activity coefficients of propane, propene, butane, 2-methylpropane, 1-butene, 2-methylpropene, trans-2-butene, cis-2-butene, 1,3-butadiene, dimethyl ether, chloroethane, and 1,1-difluoroethane in 2-propen-1-ol. Journal of Chemical \& Engineering Data 50 (6), 2045-2048.
Miyano, Y. (2005b). Henry's law constants and infinite dilution activity coefficients of propane, propene, butane, isobutane, 1-butene, isobutene, trans-2-butene, and 1,3-butadiene in 2-methyl-1-butanol, 3-methyl-1-butanol, and 2-methyl-2-butanol. Journal of Chemical \& Engineering Data 50 (1), 211-215.

Miyano, Y. (2005c). Henry's law constants and infinite dilution activity coefficients of propane, propene, butane, isobutane, 1-butene, isobutene, trans-2-butene, and 1,3-butadiene in 1-pentanol, 2-pentanol, and 3-pentanol. Journal of Chemical Thermodynamics 37 (5), 459-465.

Miyano, Y., Mitsuoka, O., Ikeda, H., Ouchi, A., Ono, T., Tsuchida, K., \& Tateishi, Y. (2005d). Henry's law constants and infinite dilution activity coefficients of propane, propene, butane, 2-methylpropane, 1-butene, 2-methylpropene, trans-2-butene, cis-2-butene, 1,3-butadiene, dimethyl ether, chloroethane, and 1,1-difluoroethane in 2-methyl-3-buten-2-ol and 3-methyl-3-buten-1-ol. Journal of Chemical \& Engineering Data 50 (6),2106-2111.

Miyano, Y., Fujihara, I., \& Sato, K. (2006a). Henry's law constants of propane, propene, butane, and 2-methylpropane in methanol at 374-490 K. Fluid Phase Equilibria 240 (1), 56-62.

Miyano, Y., Kobashi, T., Shinjo, H., Kumada, S., Watanabe, Y., Niya, W., \& Tateishi, Y. (2006b). Henry's law constants and infinite dilution activity coefficients of cis-2-butene, dimethylether, chloroethane, and 1,1-difluoroethane in methanol, 1-propanol, 2-propanol, 1-butanol, 2-butanol, isobutanol, tert-butanol, 1-pentanol, 2-pentanol, 3 - pentano $1,2-$ met hy $1-1-b u t a n o l$, 3-methyl-1-butanol, and 2-methyl-2-butanol. Journal of Chemical Thermodynamics 38 (6), 724-731.

Mullins, M., Rogers, T., \& Loll, A. (1998). Estimation of Henry's constants for aqueous systems at elevated temperatures. Fluid Phase Equilibria 150-151, 245-253. 
Murad, S., \& Gupta, S. (2000). A simple molecular dynamics simulation for calculating Henry's constant and solubility of gases in liquids. Chemical Physics Letters 319(1-2), 60-64.

Murad, S., \& Gupta, S. (2001). Molecular dynamics simulation for Henry's constant of oxygen in benzene. Fluid Phase Equilibria 187-188, 29-37.

Orbey, H., \& Sandler, S. I. (1998). Modeling vapor-liquid equilibria. New-York:Cambridge University Press.

Peng, D.-Y., \& Robinson, D. B. (1976). A new two-constant equation of state. Industrial \& Engineering Chemistry Fundamentals 15 (1), 59-64.

Prausnitz, J. M., Lichtenthaler, R. M., \& Gomes de Azevedo, E. (2000). Termodinámica molecular de los equilibrios de fases, 3ra ed. Madrid:PrenticeHall.

Redlich, O., \& Kwong, J. N. S. (1949). On the thermodynamics of solutions. V. An equation of state. Fugacities of gaseous solutions. Chemical Reviews 44(1), 233-244.

Ronze, D., Fongarland, P., Pitault, I., \& Forissier, M. (2002). Hydrogen solubility in straight run gasoil. Chemical Engineering Science 57 (4), 547-553.

Schnabel, T., Vrabec, J., \& Hasse, H. (2005). Henry's law constants of methane, nitrogen, oxygen and carbon dioxide in ethanol from 273 to $498 \mathrm{~K}$ : prediction from molecular simulation. Fluid Phase Equilibria 233 (2), 134-143.
Sieder, G., \& Maurer, G. (2004). An extension of the Peng-Robinson equation of state for the correlation and prediction of high-pressure phase equilibrium in systems containing supercritical carbon dioxide and a salt. Fluid Phase Equilibria $225,85-99$.

Soave, G. (1972). Equilibrium constants from a modified Redlich-Kwong equation of state. Chemical Engineering Science 27(6), 1197-1203.

Widom, B. (1963). Some topics in the theory of fluids. Journal of Chemical Physics 39 (11), 2808-2812.

Wong, D. S. H., Orbey, H., \& Sandler, S. I. (1992). Equation of state mixing rule for nonideal mixtures using available activity coefficient model parameters and that allows extrapolation over large ranges of temperature and pressure. Industrial \& Engineering Chemistry Research 31 (8), 2033-2039.

Wu, C., Li, X., Dai, J., \& Sun, H. (2005). Prediction of Henry's law constants of small gas molecules in liquid ethylene oxide and ethanol using force field methods. Fluid Phase Equilibria 236 (1-2), 66-77.

Zhang, L., \& Siepmann, J. I. (2006). Direct calculation of Henry's law constants from Gibbs ensemble Monte Carlo simulations: nitrogen, oxygen, carbon dioxide and methane in ethanol. Theoretical Chemistry Accounts 115 (5), 391-397.

Zhi, Y., \& Lee, H. (2002). Fallibility of analytic roots of cubic equations of state in low temperature region. Fluid Phase Equilibria 201 (2), 287-294. 